\title{
CATENOID-LIKE SOLUTIONS FOR THE MINIMAL SURFACE EQUATION
}

\author{
JENn-FAng Hwang
}

Let $\Omega \subset \mathbb{R}^{2}$ be an unbounded domain with width of polynomial growth and let $u$ satisfy the minimal surface equation in $\Omega$. We find out an upper bound function for $u$ and give an example to illustrate that the upper bound function obtained here is approximately optimal. In fact, the graph of the upper bound function is a generalization of a catenoid.

\section{Introduction.}

Consider the minimal surface equation in $\mathbb{R}^{2}$

$$
\operatorname{div} T u=0
$$

where

$$
T u=\frac{D u}{\sqrt{1+|D u|^{2}}} \quad \text { and } \quad D u=\left(u_{x}, u_{y}\right) .
$$

In 1965, Nitsche [5] announced the following result: "Let $\Omega_{\alpha} \subset \mathbb{R}^{2}$ be a sector with angle $0<\alpha<\pi$. If $u$ satisfies the minimal surface equation with vanishing boundary value in $\Omega_{\alpha}$, then $u \equiv 0$ ". Hwang extends this result in [3], [4] and proves that, in an unbounded domain $\Omega$ properly contained in the half plane in $\mathbb{R}^{2}$, if $u$ satisfies the minimal surface equation, then, the growth property of $u$ is determined completely by the shape of $\Omega$ and the boundary value of $u$. In this respect, the Phragmén-Lindelöf theorem for the minimal surface equation is better than that for the Laplace equation; (indeed, if $u$ satisfies the Laplace equation in an unbounded domain $\Omega$, the growth property of $u$ cannot be determined completely by the shape of $\Omega$ and the boundary data of $u$ alone [8]).

One of the results in [4] is the following:

Theorem 1. Let $\Omega_{m}=\left\{(x, y) \in \mathbb{R}^{2} \mid-y^{m}<x<y^{m}, y>0\right\}$, where $m$ is a constant, $m \geq 1$. If $u$ satisfies the minimal surface equation in $\Omega_{m}$ with vanishing boundary value, then

$$
u \leq \sqrt{1-\frac{1}{m}} \sqrt{y^{2 m}-x^{2}} \quad \text { in } \quad \Omega_{m} .
$$


The estimate in Theorem 1, however, is not optimal. In $\S 2$ of this paper, we shall try to find out the optimal upper bound function for solutions of (1) in $\Omega_{m}$. Then, in $\S 3$, we shall give an example to illustrate that the upper bound function obtained in $\S 2$ is approximately optimal. The crucial point of this paper is to approximate the solution of (1) in $\Omega_{m}$ with vanishing boundary value by the so-called catenoid-like solutions (which will be introduced in (3) below).

\section{Catenoid-like solutions.}

Henceforth, we shall denote $\Omega_{m}$ as the domain

$$
\left\{(x, y) \in \mathbb{R}^{2} \mid-y^{m}<x<y^{m}, y>0\right\}
$$

in $\mathbb{R}^{2}$, where $m$ is a constant, $m \geq 1$.

We first observe that the upper bound function in Theorem 1 is

$$
u \leq \sqrt{1-\frac{1}{m}} \sqrt{y^{2 m}-x^{2}}=y^{m} \sqrt{1-\frac{1}{m}} \sqrt{1-\left(\frac{x}{y^{m}}\right)^{2}} \quad \text { in } \quad \Omega_{m} .
$$

This suggests us to consider comparison functions of the following form

$$
F=y^{m} h\left(\frac{x}{y^{m}}\right)
$$

or, even more generally,

$$
F=f(y) h\left(\frac{x}{f(y)}\right)
$$

For such a function $F$, each cross-section $y=$ constant has a similar shape and the graph of $F$ is therefore a generalization of a catenoid; thus, we name such a function $F$ as a catenoid-like solution.

We shall proceed to show the following result:

Theorem 2. Let $f \in C^{2}\left(\mathbb{R}^{+}\right) \cap C^{0}\left(\overline{\mathbb{R}^{+}}\right)$, where $\mathbb{R}^{+}=(0, \infty) \subset \mathbb{R}$, and let $h \in C^{2}((-1,1)) \cap C^{1}([-1,1])$. Suppose that $F=f(y) h\left(\frac{x}{f(y)}\right)$. Then

$$
\begin{aligned}
\operatorname{div} T F= & \left(1+|D F|^{2}\right)^{-\frac{3}{2}} \frac{f^{\prime 2}}{f} \\
& \cdot\left((1-p)\left(h-h^{\prime} t\right)\left(h^{\prime 2}+1\right)+h^{\prime \prime}\left(h^{2}+t^{2}\right)+\frac{h^{\prime \prime}}{\left(f^{\prime}\right)^{2}}\right)
\end{aligned}
$$


where $t=\frac{x}{f(y)}$ and $1-p=\frac{f f^{\prime \prime}}{\left(f^{\prime}\right)^{2}}$.

Proof. By a direct calculation, we have

$$
\begin{aligned}
& t_{x}=\frac{1}{f(y)} \\
& t_{y}=\frac{-f^{\prime}(y)}{f^{2}(y)} x=-\frac{f^{\prime}(y)}{f(y)} t,
\end{aligned}
$$

and then

$$
\begin{aligned}
& F_{x}=f(y) h^{\prime}(t) t_{x}=h^{\prime}(t), \\
& F_{y}=f^{\prime}(y) h(t)+f(y) h^{\prime}(t) t_{y}=f^{\prime}(y)\left(h(t)-h^{\prime}(t) t\right) .
\end{aligned}
$$

Thus

$$
\begin{aligned}
F_{x x} & =h^{\prime \prime}(t) t_{x}=\frac{h^{\prime \prime}(t)}{f(y)}, \\
F_{x y} & =-\frac{f^{\prime}(y)}{f(y)} t h^{\prime \prime}(t), \\
F_{y y} & =f^{\prime \prime}(y)\left(h(t)-t h^{\prime}(t)\right)+f^{\prime}(y)\left(h^{\prime}(t)-t h^{\prime \prime}(t)-h^{\prime}(t)\right)\left(-\frac{f^{\prime}(y)}{f(y)} t\right) \\
& =f^{\prime \prime}(y)\left(h(t)-t h^{\prime}(t)\right)+\frac{f^{\prime 2}(y)}{f(y)} t^{2} h^{\prime \prime}(t) .
\end{aligned}
$$

Hence

$$
\begin{aligned}
F_{x x}+F_{y y} & =f^{\prime \prime}\left(h-t h^{\prime}\right)+\frac{f^{\prime 2}}{f} t^{2} h^{\prime \prime}+\frac{h^{\prime \prime}}{f} \\
& =(1-p) \frac{f^{\prime 2}}{f}\left(h-t h^{\prime}\right)+\frac{f^{\prime 2}}{f} t^{2} h^{\prime \prime}+\frac{h^{\prime \prime}}{f} \\
& =\frac{f^{\prime 2}}{f}\left((1-p)\left(h-t h^{\prime}\right)+t^{2} h^{\prime \prime}+\frac{h^{\prime \prime}}{f^{\prime 2}}\right) .
\end{aligned}
$$

And

$$
\begin{aligned}
& F_{x}^{2} F_{y y}-2 F_{x} F_{y} F_{x y}+F_{y}^{2} F_{x x} \\
& =h^{\prime 2}\left(f^{\prime \prime}\left(h-t h^{\prime}\right)+\frac{f^{\prime 2}}{f} t^{2} h^{\prime \prime}\right)-2 f^{\prime} h^{\prime}\left(h-t h^{\prime}\right)\left(-\frac{f^{\prime}}{f} t h^{\prime \prime}\right)+f^{\prime 2}\left(h-t h^{\prime}\right)^{2} \frac{h^{\prime \prime}}{f} \\
& =(1-p) \frac{f^{\prime 2}}{f} h^{\prime 2}\left(h-t h^{\prime}\right)+\frac{f^{\prime 2}}{f} t^{2} h^{\prime 2} h^{\prime \prime}+2 \frac{f^{\prime 2}}{f}\left(h-t h^{\prime}\right) t h^{\prime} h^{\prime \prime}+\frac{f^{\prime 2}}{f} h^{\prime \prime}\left(h-t h^{\prime}\right)^{2} \\
& =\frac{f^{\prime 2}}{f}\left((1-p) h^{\prime 2}\left(h-t h^{\prime}\right)+h^{\prime 2} h^{\prime \prime} t^{2}+2 h^{\prime}\left(h-t h^{\prime}\right) t h^{\prime \prime}+h^{\prime \prime}\left(h-t h^{\prime}\right)^{2}\right) \\
& =\frac{f^{\prime 2}}{f}\left((1-p) h^{\prime 2}\left(h-t h^{\prime}\right)+h^{2} h^{\prime \prime}\right) .
\end{aligned}
$$


Finally, we obtain, by adding the last two identities,

$$
\begin{aligned}
\operatorname{div} T F & =\left(1+|D F|^{2}\right)^{-\frac{3}{2}}\left(\left(1+F_{x}^{2}\right) F_{y y}-2 F_{x} F_{y} F_{x y}+\left(1+F_{y}{ }^{2}\right) F_{x x}\right) \\
& =\left(1+|D F|^{2}\right)^{-\frac{3}{2}} \frac{f^{\prime 2}}{f}\left((1-p)\left(h-t h^{\prime}\right)\left(1+h^{\prime 2}\right)+h^{\prime \prime}\left(h^{2}+t^{2}\right)+\frac{h^{\prime \prime}}{f^{\prime 2}}\right),
\end{aligned}
$$

as desired.

In the special case where

$$
f(y)=y^{m}, 1 \leq m<\infty, m=\text { a constant, }
$$

we have

$$
p=p(f)=\frac{1}{m},
$$

which is also a constant; moreover, in the bracket in the expression of $\operatorname{div} T F$, the order of growth of the term $\frac{h^{\prime \prime}}{f^{\prime 2}}$ is lower than that of the others. Thus, to obtain an optimal comparison function, we may first consider the equation

$$
\left(1-\frac{1}{m}\right)\left(h_{m}-t h_{m}^{\prime}\right)\left(1+{h_{m}^{\prime}}^{2}\right)+h_{m}^{\prime \prime}\left(h_{m}^{2}+t^{2}\right)=0
$$

where the domain of definition is $[-1,1]$, throughout which we require that

$$
h_{m}(t)>0 \quad \text { for } \quad t \in(-1,1) .
$$

We specify one of the initial conditions as

$$
h_{m}(-1)=0,
$$

and now proceed to determine the other initial data $h_{m}^{\prime}(-1)$. For this, we note that, (4) yields

$$
\frac{h_{m}^{\prime \prime}}{1+{h_{m}^{\prime}}^{2}}=\left(1-\frac{1}{m}\right) \frac{t h_{m}^{\prime}-h_{m}}{t^{2}+h_{m}{ }^{2}}
$$

that is,

$$
\left(\tan ^{-1} h_{m}^{\prime}\right)^{\prime}=-\left(1-\frac{1}{m}\right)\left(\tan ^{-1} \frac{t}{h_{m}}\right)^{\prime}
$$

which yields

$$
\left.\tan ^{-1} h_{m}^{\prime}\right|_{0} ^{t}=-\left.\left(1-\frac{1}{m}\right) \tan ^{-1} \frac{t}{h_{m}}\right|_{0} ^{t} .
$$


Thus, imposing an additional condition that

$$
h_{m}^{\prime}(0)=0,
$$

we have

$$
\tan ^{-1} h_{m}^{\prime}=-\left(1-\frac{1}{m}\right) \tan ^{-1} \frac{t}{h_{m}}
$$

or, equivalently,

$$
h_{m}^{\prime}(t)=-\tan \left(\left(1-\frac{1}{m}\right) \tan ^{-1} \frac{t}{h_{m}}\right) .
$$

Since (5) holds for all $t \in(-1,1)$, we note, by (8) and the assumed continuity of $h$, that

$$
h_{m}^{\prime}(-1)=-\tan \left(\left(1-\frac{1}{m}\right)\left(-\frac{\pi}{2}\right)\right)=\tan \left(\left(1-\frac{1}{m}\right) \frac{\pi}{2}\right),
$$

which is the second initial condition of (4). We note that (7) and (8) yield

$$
h_{m}(-t)=h_{m}(t)
$$

for all $t \in(-1,1)$. Hence,

$$
\begin{cases}h_{m}(1)=0, & \\ h_{m}^{\prime}(t) \geq 0, & \text { for }-1 \leq t \leq 0, \\ h_{m}^{\prime}(t) \leq 0, & \text { for } \quad 0 \leq t \leq 1 .\end{cases}
$$

From this, it follows that:

Lemma 1. $h_{m}\left(t_{2}\right) \leq h_{m}\left(t_{1}\right)$, whenever $0 \leq\left|t_{1}\right| \leq\left|t_{2}\right| \leq 1$.

Also, (10) yields

$$
h_{m}-t h_{m}^{\prime} \geq 0, \quad \text { for } \quad-1 \leq t \leq 1 \text {, }
$$

and hence, by virtue of (4),

$$
h_{m}^{\prime \prime} \leq 0, \quad \text { for } \quad-1 \leq t \leq 1 .
$$

We may also note that, for a constant $q$ with $1 \leq q<m<\infty$, since $h_{m}$ and $h_{q}$ are both solutions of (4) with

$$
h_{m}( \pm 1)=h_{q}( \pm 1)=0
$$


while

$$
h_{m}^{\prime}(-1)=\tan ^{-1}\left(\left(1-\frac{1}{m}\right) \frac{\pi}{2}\right)>\tan ^{-1}\left(\left(1-\frac{1}{q}\right) \frac{\pi}{2}\right)=h_{q}^{\prime}(-1),
$$

therefore

$$
h_{m}(t)>h_{q}(t)
$$

for all $t \in(-1,1)$.

In general, we cannot write out explicitly a solution of the equation (8) with initial data (6). However, for some specific $m$, the solution can be explicitly written out. For example, for $m=2$, we have

$$
h_{2}(t)=\frac{1-t^{2}}{2} \text {. }
$$

In case that $f(y)=e^{y}$ in (3), we have $p(f)=1-\frac{f f^{\prime \prime}}{f^{\prime 2}}=0$. Thus, we may formally define $m=\infty$ and $\frac{1}{m}=0$, substituting this into (8) to obtain

$$
h_{\infty}=\sqrt{1-t^{2}}
$$

since $h_{\infty}(-1)=0$ and $h_{\infty}^{\prime}(-1)=\infty$, we have

$$
h_{m}(t)<h_{\infty}(t)
$$

for every $t \in(-1,1)$ and every constant $1 \leq m<+\infty$.

We are now in a position to prove the following Main Theorem of this paper.

Theorem 3. Let $1 \leq m<\infty$ be a constant and let

$$
\Omega \subset\left\{(x, y) \in \mathbb{R}^{2} \mid-a y^{m}<x<a y^{m}, y>0\right\},
$$

where $a$ is a positive constant. Let $u \in C^{0}(\bar{\Omega}) \cap C^{2}(\Omega)$. Suppose that

$$
\begin{cases}\text { (i) } \operatorname{div} T u \geq 0 & \text { in } \quad \Omega . \\ \text { (ii) } u \leq a y^{m} h_{m}\left(\frac{x}{a y^{m}}\right) & \text { on } \partial \Omega .\end{cases}
$$

Then

$$
u \leq a y^{m} h_{m}\left(\frac{x}{a y^{m}}\right) \quad \text { in } \quad \Omega .
$$

\section{Remark.}

(i) In this theorem, no growth condition on $u$ is imposed. 
(ii) When $f(y)=e^{y}, m=\infty$ and Theorem 3 still holds with $a y^{m}$ replaced by $e^{y}$.

(Cf. [4])

Proof. For every positive constant $\epsilon$, by a direct computation, we have

$$
a y^{m} \leq a\left(y+\frac{\epsilon}{m}\right)^{m+\epsilon}, \quad \text { for every } \quad y>0 .
$$

Set

$$
G_{\epsilon}=a\left(y+\frac{\epsilon}{m}\right)^{m+\epsilon} h_{m+\epsilon}\left(\frac{x}{a\left(y+\frac{\epsilon}{m}\right)^{m+\epsilon}}\right) .
$$

Since

$$
\begin{aligned}
& \left\{(x, y) \in \mathbb{R}^{2} \mid-a\left(y+\frac{\epsilon}{m}\right)^{m+\epsilon}<x<a\left(y+\frac{\epsilon}{m}\right)^{m+\epsilon}, y>0\right\} \\
& \supset\left\{(x, y) \in \mathbb{R}^{2} \mid-a y^{m}<x<a y^{m}, y>0\right\}
\end{aligned}
$$

by Theorem 2, (4) and (11), we have

$$
\operatorname{div} T G_{\epsilon} \leq 0 \quad \text { in } \quad \Omega .
$$

Also, on the boundary $\partial \Omega$ of $\Omega$,

$$
\begin{aligned}
u & \leq a y^{m} h_{m}\left(\frac{x}{a y^{m}}\right) \quad \text { (by assumption) } \\
& \leq a\left(y+\frac{\epsilon}{m}\right)^{m+\epsilon} h_{m}\left(\frac{x}{a\left(y+\frac{\epsilon}{m}\right)^{m+\epsilon}}\right) \quad \text { (by (14) and (10)) } \\
& =G_{\epsilon} .
\end{aligned}
$$

Moreover, by [4, Theorem 2.10],

$$
u \leq \sqrt{1-\frac{1}{m}} a y^{m} h_{\infty}\left(\frac{x}{a y^{m}}\right) \quad \text { in } \quad \Omega ;
$$

hence, by (13),

$$
u \leq \sqrt{1-\frac{1}{m}} a y^{m} \quad \text { in } \quad \Omega .
$$

Note that

$$
\lim _{y \rightarrow \infty} \frac{a y^{m}}{a\left(y+\frac{\epsilon}{m}\right)^{m+\epsilon}}=0
$$


it is easy to see that

$$
\sqrt{1-\frac{1}{m}} a y^{m} \leq G_{\epsilon} \quad \text { for } y \text { sufficiently large. }
$$

An application of the maximum principle, together with (15), (16), and (17), yields that

$$
u \leq G_{\epsilon} \quad \text { in } \quad \Omega .
$$

Letting $\epsilon \rightarrow 0$, the theorem follows immediately.

\section{Examples to illustrate that the estimate in Theorem 3 is approximately optimal.}

Let $m, 1<m<\infty$, be a constant. In this section, we shall construct a solution $u$ of the minimal surface equation in a domain $\Omega$ with

$$
\lim _{y \rightarrow \infty} \frac{\left|\Gamma_{y}\right|}{y^{m}}=2 \quad\left(\text { where } \quad \Gamma_{y_{0}}=\Omega \cap\left\{y=y_{0}\right\}, \quad\left|\Gamma_{y}\right| \text { is the length of } \Gamma_{y}\right. \text { ) }
$$

such that

$$
\lim _{y \rightarrow \infty}\left(\max _{\Gamma_{y}} \frac{u}{y^{m}}\right)=h_{m}(0)
$$

This shall illustrate that the estimate in Theorem 3 is approximately optimal.

The discussion will be divided into two cases seperately, namely

Case 1: $\quad+\infty>m>1.5$

Case 2: $\quad 1.5 \geq m>1$.

We consider first:

Case 1. $\quad+\infty>m>1.5$.

Let $f(y)=y^{m}+y^{0.5} \quad$ where $\quad y>0$.

Then

$$
\begin{aligned}
& 1-p(f) \\
& =\frac{f f^{\prime \prime}}{f^{\prime 2}} \\
& =\frac{\left(y^{m}+y^{0.5}\right)\left(m(m-1) y^{m-2}-0.25 y^{-1.5}\right)}{\left(m y^{m-1}+0.5 y^{-0.5}\right)^{2}} \\
& =\left(1-\frac{1}{m}\right)+\left(f^{\prime}\right)^{-2}\left[(m-1.5)(m-0.5) y^{m-1.5}-0.5\left(1-\frac{0.5}{m}\right) y^{-1}\right] .
\end{aligned}
$$

Let

$$
F(x, y)=f(y) h_{m}\left(\frac{x}{f(y)}\right)
$$


where $y>0,-f(y)<x<f(y)$. Now, setting $t=\frac{x}{f(y)}$, we have

$$
\begin{aligned}
\operatorname{div} T F= & \left(1+|D F|^{2}\right)^{-\frac{3}{2}} \frac{\left(f^{\prime}\right)^{2}}{f} \\
& \cdot\left((1-p(f))\left(h_{m}-t h_{m}^{\prime}\right)\left(1+{h_{m}^{\prime}}^{2}\right)+h_{m}^{\prime \prime}\left(t^{2}+{h_{m}}^{2}\right)+\frac{h_{m}^{\prime \prime}}{\left(f^{\prime}\right)^{2}}\right) .
\end{aligned}
$$

Hence, by (4) and (18), we have

$$
\begin{aligned}
\operatorname{div} T F= & \left(1+|D F|^{2}\right)^{-\frac{3}{2}} \frac{\left(f^{\prime}\right)^{2}}{f} \\
& \cdot\left(\left(\left(f^{\prime}\right)^{-2}\left[(m-1.5)(m-0.5) y^{m-1.5}-0.5\left(1-\frac{0.5}{m}\right) y^{-1}\right]\right)\right. \\
& \left.\cdot\left(h_{m}-t h_{m}^{\prime}\right)\left(1+{h_{m}^{\prime}}^{2}\right)+\frac{h_{m}^{\prime \prime}}{\left(f^{\prime}\right)^{2}}\right) .
\end{aligned}
$$

By (4) again, we have

$$
\left(1+{h_{m}^{\prime}}^{2}\right)\left(h_{m}-t h_{m}^{\prime}\right)=-\frac{m}{m-1} h_{m}^{\prime \prime}\left(h_{m}^{2}+t^{2}\right)
$$

Thus

$$
\begin{aligned}
& \operatorname{div} T F=\left(1+|D F|^{2}\right)^{-\frac{3}{2}} \frac{\left(-h_{m}^{\prime \prime}\right)}{f} \\
& \cdot\left(\frac{m}{m-1}\left((m-1.5)(m-0.5) y^{m-1.5}-0.5\left(1-\frac{0.5}{m}\right) y^{-1}\right)\left(h_{m}{ }^{2}+t^{2}\right)-1\right) .
\end{aligned}
$$

Hence, as $h_{m}{ }^{2}+t^{2}$ is bounded below by a positive constant depending only on $m$, (11) yields a positive number $y_{1}$, such that

$$
\operatorname{div} T F \geq 0, \quad \text { for all } y \geq y_{1} .
$$

Next, let

$$
G=f(y) h_{m+\epsilon}\left(\frac{x}{f(y)}\right),
$$

where $\epsilon$ is a positive constant. By the same calculation, we obtain

$$
\begin{aligned}
\operatorname{div} T G=\left(1+|D G|^{2}\right)^{-\frac{3}{2}} \frac{\left(-h_{m+\epsilon}^{\prime \prime}\right)}{f} & \cdot\left(\left(\frac { m + \epsilon } { ( m + \epsilon ) - 1 } \left((m-1.5)(m-0.5) y^{m-1.5}\right.\right.\right. \\
& \left.\left.-0.5\left(1-\frac{0.5}{m}\right) y^{-1}\right)\left(h_{m+\epsilon}^{2}+t^{2}\right)-1\right) \\
& \left.+\frac{m+\epsilon}{(m+\epsilon)-1}\left(\frac{1}{m+\epsilon}-\frac{1}{m}\right)\left(h_{m+\epsilon}^{2}+t^{2}\right)\left(f^{\prime}\right)^{2}\right) .
\end{aligned}
$$


Since $f^{\prime}=m y^{m-1}+0.5 y^{-0.5}$, it is easy to see that there exists a positive constant $y_{2}$, determined by on $m$ and $\epsilon$, such that $y_{2}>y_{1}$ and

$$
\operatorname{div} T G \leq 0, \quad \text { for all } y \geq y_{2} .
$$

To summarize up, we have constructed the functions $F$ and $G$ as the lower and upper barriers, respectively; that is, we have

$$
\begin{cases}\operatorname{div} T G \leq 0 \leq \operatorname{div} T F & \text { in } \Omega^{\prime} \\ G=F=0 & \text { on } \partial \Omega^{\prime} \backslash\left\{y=y_{2}\right\} \\ G \geq F & \text { in } \Omega^{\prime},\end{cases}
$$

where $\Omega^{\prime}=\left\{(x, y) \in \mathbb{R}^{2} \mid-\left(y^{m}+y^{0.5}\right)<x<y^{m}+y^{0.5}, y>y_{2}\right\}$. As Perron's method can be adopted here (cf. [6, p. 593-600]), this gives us a function $u^{\prime}$ defined in $\Omega^{\prime}$ such that

$$
\begin{cases}\operatorname{div} T u^{\prime}=0 & \text { in } \quad \Omega^{\prime} \\ G \geq u^{\prime} \geq F & \text { in } \quad \Omega^{\prime} \\ u^{\prime}=0 & \text { on } \quad \partial \Omega^{\prime} \backslash\left\{y=y_{2}\right\} .\end{cases}
$$

Set

$$
u=u^{\prime}-\max _{\left\{y=y_{2}\right\}} u^{\prime}
$$

and

$$
\Omega^{\prime \prime}=\left\{(x, y) \in \Omega^{\prime} \mid u^{\prime}(x, y)>\max _{\left\{y=y_{2}\right\}} u^{\prime}, y>y_{2}\right\} .
$$

Then $u=0$ on $\partial \Omega^{\prime \prime}$. It is easy to see thet $\Omega^{\prime \prime}$ contains $(0, y)$ for all sufficiently large $y$, and $\Omega^{\prime \prime}$ contains $\left\{(x, y) \mid-y^{m}<x<y^{m}, \quad y>y_{0}\right\}$ where $y_{0}$ is sufficiently large. To see that $u$ is our desired optimal solution, it remains to show that the behaviour of $u$ near infinity is asymptotic to that of the function $y^{m} h_{m}\left(\frac{x}{y^{m}}\right)$. To do so, we may define

$$
H(x, y)=(y+1)^{m} h_{m}\left(\frac{x}{(y+1)^{m}}\right)+\max _{\left\{y=y_{2}\right\}} u^{\prime}(x, y)
$$

then, as

$$
(y+1)^{m} \geq y^{m}+y^{0.5},
$$

we have $H(x, y)$ defined in $\Omega^{\prime}$ and $H \geq u^{\prime}$ on $\partial \Omega^{\prime}$. Hence, by Theorem 3,

$$
H \geq u^{\prime} \quad \text { in } \quad \Omega^{\prime} .
$$

This, together with the inequality that

$$
u^{\prime} \geq F \quad \text { in } \quad \Omega^{\prime}
$$


gives us the desired estimate of the behaviour of $u^{\prime}$ and $u$ near the infinity.

Next, we consider

Case 2. $1.5 \geq m>1$.

Let $f(y)=y^{m}-y^{\beta}$, for all $y>0$, where $\beta=\frac{m+1}{2}$. Then

$$
\begin{aligned}
& 1-p(f) \\
& =1-\frac{1}{m}+\left(f^{\prime}\right)^{-2}\left(-(m-\beta-1)(m-\beta) y^{m+\beta-2}-\beta\left(1-\frac{\beta}{m}\right) y^{2 \beta-2}\right),
\end{aligned}
$$

in which we may note that $-(m-\beta-1)(m-\beta)>0$ and $m+\beta-2>0$.

Let

$$
F(x, y)=f(y) h_{m}\left(\frac{x}{f(y)}\right)
$$

and

$$
G(x, y)=f(y) h_{m+\epsilon}\left(\frac{x}{f(y)}\right)
$$

where $\epsilon$ is a positive constant. By the same reasoning as in Case 1, there exists a positive constant $y_{3}$ such that, in

$$
\Omega_{3}=\left\{(x, y) \in \mathbb{R}^{2} \mid-f(y)<x<f(y), y>y_{3}\right\},
$$

we have

$$
\operatorname{div} T F \geq 0
$$

and

$$
\operatorname{div} T G \leq 0
$$

These again give us a function $u$, defined in $\Omega_{3}$, such that

$$
\begin{cases}\operatorname{div} T u=0 & \text { in } \quad \Omega_{3} \\ F \leq u \leq G & \text { in } \quad \Omega_{3} \\ u=0 & \text { on } \partial \Omega_{3} \backslash\left\{y=y_{3}\right\} .\end{cases}
$$

Moreover, since $y^{m}>y^{m}-y^{\beta}=f(y)$, an upper bound for $u$ is

$$
y^{m} h_{m}\left(\frac{x}{y^{m}}\right)+\max _{\left\{y=y_{3}\right\}} u \text {. }
$$

Thus, the function

$$
u^{\prime}=u-\max _{\left\{y=y_{3}\right\}} u
$$


is what looked for.

Acknowledgments. The author would like to express his gratitude to Professor Jin-Tzu Chen for bringing this problem to his attention, and he would like to thank the referee for many helpful comments and suggestions.

\section{References}

[1] R. Finn, Equilibrium capillary surfaces, Springer-Verlag, New York-Berlin-Heidelberg-Tokyo, 1986.

[2] D. Gilbarg and N.S. Trudinger, Elliptic partial differential equations of second order, (second edition), Springer-Verlag, Berlin-Heidelberg-New York-Tokyo, 1983.

[3] J. Hwang, Phragmén-Lindelöf theorem for the minimal surface equation, Proc. Amer. Math. Soc., 104 (1988), 825-828.

[4] Growth property for the minimal surface equation in unbounded domains, Proc. Amer. Math. Soc., 121 (1994), 1027-1037.

[5] J.C.C. Nitsche, On new results in the theory of minimal surface, Bull. Amer. Math. Soc., 71 (1965), 195-270.

[6] _ Vorlesungen über Minimalflächen, Springer-Verlag, Berlin-Heidelberg-New York, 1975.

[7] R. Osserman, A survey of minimal surfaces, Van Nostrand-Reinhold, New York, 1969 .

[8] M.H. Protter and H.F. Weinberger, Maximum principles in differential equations, Prentice-Hall, Englewood Cliffs, N.J., 1967.

Received January 15, 1996 and revised October 9, 1996. This research was partially supported by Grant NSC84-2121-M-001-013.

ACADEMia Sinica

TAIPEI 11529

TAIWAN, R.O.C.

E-mail address: MAJFH@ccvax.sinica.edu.tw 